\title{
Next-generation sequencing as an advanced supplementary tool for the diagnosis of pathogens in lower respiratory tract infections: An observational trial in Xi'an, China
}

\author{
JIE SHAO $^{1}$, AMIRA HASSOUNA $^{2,3}$, YAQIN WANG $^{1}$, RUIRUI ZHANG $^{1}$, LIFANG ZHEN $^{1}$, \\ RUIDAN LI $^{1}$, MINGLI CHEN ${ }^{1}$, CHENGJIE LIU ${ }^{1}$, XIANGYE WANG ${ }^{1}$, MINGMING ZHANG $^{1}$, \\ PENG WANG $^{1}$, SHENGHUA YUAN $^{1}, \mathrm{JIE} \mathrm{CHEN}^{4}$ and JUN LU ${ }^{2-8}$
}

\begin{abstract}
${ }^{1}$ Norinco 521 Hospital, Xi'an, Shaanxi 710065, P.R. China; ${ }^{2}$ School of Public Health and Interdisciplinary Studies, Faculty of Health and Environmental Sciences, Auckland University of Technology, Auckland 0622, New Zealand; ${ }^{3}$ Department of Medical Biochemistry and Molecular Biology, Faculty of Medicine, Cairo University, Cairo 12613, Egypt;

${ }^{4}$ Guangzhou Sagene Biotech Co., Ltd., Guangzhou, Guangdong 510320; ${ }^{5}$ College of Food Science and Technology, Nanchang University, Nanchang, Jiangxi 330031; ${ }^{6}$ College of Life Sciences and Oceanography, Shenzhen University, Shenzhen, Guangdong 518071, P.R. China; ${ }^{7}$ School of Science, Faculty of Health and Environmental Sciences, Auckland University of Technology; ${ }^{8}$ Maurice Wilkins Centre for Molecular Discovery, Auckland 1142, New Zealand
\end{abstract}

Received October 1, 2021; Accepted December 7, 2021

DOI: 10.3892/br.2021.1497

\begin{abstract}
The application of next-generation sequencing (NGS) in routine clinical analysis is still limited. The significance of NGS in the identification of pathogens of lower respiratory tract infection should be assessed as part of routine clinical bacterial examinations and chest imaging results. In the present study, the alveolar lavage fluid samples of 30 patients ( 25 males and 5 females, aged 19-92 years old, with a median age of 62) were examined by routine bacterial culture and NGS, and the results of pathogen detection and identification were compared. Chest imaging showed consolidation in all 30 patients (100\%), and pleural effusion in 13 of the 30 patients $(43.33 \%)$. The routine bacterial culture of the lavage solution was only positive in 14 of the 30 patients (46.6\%), and negative in 16 patients (53.33\%). However, the positive rate of NGS test results of the lavage fluid was $100 \%$. A total of 12 cases $(40 \%)$ were completely consistent with the routine bacterial culture test, with 56 other pathogens of mixed infection detected, accounting for the short comings
\end{abstract}

Correspondence to: Dr Jie Shao, Norinco 521 Hospital, 12 Zhangba East Road, Xi'an, Shaanxi 710065, P.R. China E-mail: shaojie1001521@126.com

Professor Jun Lu, School of Public Health and Interdisciplinary Studies, Faculty of Health and Environmental Sciences, Auckland University of Technology, 90 Akoranga Drive, Auckland 0622, New Zealand

E-mail: jun.lu@aut.ac.nz

Key words: pulmonary infection, diagnosis, next-generation sequencing, bronchoalveolar lavage fluid, bacterial culture of the routine bacterial examination. Although NGS cannot distinguish between live and dead bacteria, it is still a useful detection technology for accurate diagnosis of clinical infectious diseases. It is worthy of adaptation in the clinic for more effective clinical management and treatment of the lower respiratory airway infection in addition to the routine bacterial culture testing.

\section{Introduction}

Lower respiratory tract infections (LRTIs) refer to infections that involve the respiratory tract below the level of the larynx, and includes tracheitis, bronchitis, bronchiolitis and bronchopneumonia $(1,2)$. LRTIs are the major cause of pneumonia and bronchiolitis in hospitals worldwide (1). LRTIs cause $\sim 3.5$ million deaths, as well as 79 million cases of disabilities annually (2). LRTIs are associated with high overall morbidity and mortality in adults, with increased risk in the elderly compared to young adults $(3,4)$. Children admitted with LRTIs were reported to have severe respiratory disease (5), in addition to suffering respiratory sequelae (6) and having a long recovery period (5). Among acute LRTIs, the major common cause for hospitalization of infants is bronchiolitis (7), whereas pneumonia in adults (mainly $>65$ years) and children is the most common cause of death, accounting for 3.5 million (6.1\%) deaths globally and over 1.05 million (11.3\%) in lower income countries $(8,9)$ every year.

Identification of causative organisms is essential for definitive diagnosis of infectious diseases, as well as decision making with regard to appropriate management. The gold standard method is the usage of bacterial and fungal cultures; however, it is time-consuming and not suitable for identification of unculturable bacteria. PCR and antigen tests are commonly used for viral detection; however, only 
a defined set of microorganisms can be examined (10). Although a reliable method of identification, viral isolation is also time-consuming, and is insufficiently sensitive. Performing combinations of these procedures, did not show significantly improved pathogen identification in $34-57 \%$ of paediatric patients (10), and in $13-62 \%$ of adult patients with pneumonia $(11,12)$. In the case of respiratory infection, diagnosis of specific microorganisms occurs when they are isolated from sterile materials such as bronchoalveolar lavage fluid (BALF) $(10,13,14)$. BALF is more suitable than sputum for identifying pathogens of respiratory infections, as less contamination with oral bacteria is possible during collection from the locus of infection (15). BALF is usually obtained for PCR to detect certain viruses $(16,17)$. It is to be noted that the diagnostic yield of causative pathogens from BALF in lower respiratory tract infections in immunosuppressed children was $28-68 \%$ (18). BALF is collected using a safe procedure and with a very low rate of morbidity and mortality (19). Several studies reported that the results for microbiological examination of BALF have led to alterations in the management of infections in $38.7-72.7 \%$ of patients (18-20).

LRTIs are commonly managed by treatment with antibiotics; however, clinical management of LRTIs is difficult due to antibiotic resistance caused by inappropriate use of antimicrobial agents, leading to increased hospital mortality rates, as well as excessive costs for LRTI patients (21).

As the effective selection of antibiotics is considered a considerable challenge for all physicians (22), accurate and early identification of the pathogenic bacteria is crucial for the effective selection and use of antibiotics, and may lead to improvements in the management of patients with LRTIs worldwide.

Next-generation sequencing (NGS) has been used to identify causative pathogens in various infectious diseases (23-27). It has been proven to be useful for identification of causative organisms in several diseases, such as encephalitis, hepatitis, bloodstream infection and acute myocarditis (28-31). NGS has already been used to detect pathogens from BALF samples. A recent study detected significant reads of candidate pathogens in $34 \%$ of BALF samples collected from patients with infectious as well as non-infectious diseases (32). In another study, NGS was used to detect pathogens in the BALF of patients with respiratory infection, and the results suggest that comprehensive molecular diagnostics, as well as surveillance of pathogens in BALF can be achieved using NGS (15).

In the present study, NGS technology was used to evaluate its role in accurate detection and identification of pathogens in the alveolar lavage fluid of patients with lower respiratory tract infection, and the results were compared with those of routine pathogen cultures.

\section{Materials and methods}

Patients. The present clinical observational study was approved by Norinco 521 Hospital Ethics Committee (Xi'an, China; approval no. 201901) on 9th of January 2019. Between January 2019 and December 2019 in Norinco 521 Hospital, 30 patients were recruited for this study. All were confirmed to have a pulmonary infection based on the clinical symptoms and signs, blood tests and imaging examination. The patients included 25 males and 5 females (male: female, 5:1). The age range of the patients was 19-92 years old, with a median age of 62 .

Equipment and reagents. The following equipment were used: Dynamag TM-2 Magnetic frame (Thermo Fisher Scientific, Inc.), HEMA9600 PCR Instrument (China Haidu Black Horse Medical Instrument Co.), Nextseq 550DX sequencer (Illumina, Inc.), Bioruptor GL-16 Sonication Device (Diagenode SA), $5 \mathrm{M}$ high speed refrigerated centrifuge (Shanghai Lu Xiangyi Instrument Co., Ltd.), 2100 Biological analyzer (Agilent Technologies, Inc.) and a Qubit ${ }^{\mathrm{TM}} 4$ Fluorometer Nucleic Acid analyzer (Thermo Fisher Scientific, Inc.). The following reagents were used: QIAamp Cador assay kit (cat. no. 54104; Qiagen $\mathrm{GmbH}$ ), a nucleic acid concentration assay kit (Qubit ${ }^{\mathrm{TM}}$ dsDNA HS kit, 500 Assays; cat. no. Q32854; Thermo Fisher Scientific, Inc.), a library construction kit (Sagene DNA Library kit; cat. no. Cp.000002; Sagene Biotechnology) and an NSQ 500/550 Hi Output KT V2.5 (75 CYS) sequencing kit (cat. no. 20024906; Illumina, Inc.).

Specimen collection. The clinical team was involved in talking the participating patients through the process and obtaining signed consent forms before routine tracheal examination. During the tracheal examination, the front end of the trachea was placed in the tube cavity with the most obvious pulmonary infection; $10 \mathrm{ml}$ normal saline was used for bronchial lavage. Part of the lavage fluid $(\sim 3 \mathrm{ml})$ was sent for routine bacterial culture, and $3 \mathrm{ml}$ of the lavage fluid was placed into a sterile, sealable special freezing-storage tube. After sampling, it was immediately placed in an ice box for preservation, and the whole process was preserved with dry ice and a cold storage chain before testing.

Detection process. Volumes of $1 \mathrm{ml}$ samples were collected from the subjects. In order to improve the efficiency of pathogen detection, the samples were first enriched into small volumes $(\sim 200 \mu \mathrm{l})$ and centrifuged at $12,000 \mathrm{x} \mathrm{g}$ for $10 \mathrm{~min}$ at $4^{\circ} \mathrm{C}$. Then the $200 \mu \mathrm{l}$ enriched solutions were used for nucleic acid extraction and purification using a nucleic acid extraction kit combined with magnetic beads (Sagene, Guangzhou, China) according to the manufacturer's protocol, and the metagenomic library was respectively constructed according to the protocol of the library construction kit, Nextera XT (Illumina, Inc.). According to the manufacturer's protocol, the extracted DNA was broken into 300 bp fragments different and index sequences were added. The library size and quantification were analyzed using a Qubit 4 system and Agarose $(0.8 \%$ gel) electrophoresis. After the libraries were mixed with equal amounts of the samples, high-throughput sequencing was performed on an Illumina Nextseq 550 DX sequencing platform (sequencing strategy, SE75), an FDA-approved and CE-IVD-certified sequencer. Sequencing details are listed in Table SI, and the data were obtained for subsequent bioinformatics analysis.

Bioinformatics analysis. SOAPnuke software (https://github. com/BGI-flexlab/SOAPnuke, version 1.6.0) was used to filter the data, including removing the base sequences containing sequencing joints, the base sequences consisting of ' $N$ ' 
in abundance, and the base sequences containing low quality bases reads. The filtered data were the pure base sequences. All reads were simulated from, and where relevant, aligned to the human genome (Homo sapiens version: GRCh38) using Bowtie2 (https://sourceforge. net/projects/bowtie-bio/files/bowtie2/2.3.5.1/; version 2.3.5.1).

The anthropogenic related base sequence was removed, and pathogenic microorganism database comparison was performed on the remaining base sequences after Sagene biological optimization by BWA (https://sourceforge. net/projects/bio-bwa/files/; version 0.7.15).

Appraisal of the quantitative results of pathogenic microorganisms was then performed by the senior technician of Sagene Biology Ltd., followed by the generation of a clinical test report. The patients received all the microbiological testing information. The unique pathogenic microorganism database of Guangzhou Sagene Biology was used. The database uses the NCBI database (fp://ncbi nlm.nih.gov/genomes), the Ensemble database (http://ensemblgenomes.org/), Virus Resource database (Virus get Variation Resource), JGI Fungi Porta (http://genome.jgi.doe.gov) and other authoritative microorganism databases as references. The microbial standard was used for verification and optimization.

Statistical analysis. For general clinical information, the mean \pm standard deviation, as well as the numerical range are used to describe the data. Percentages are used to the describe individual microbial detection rates in patients.

Routine bacterial culture analyses were performed, and statistical analysis was performed manually by the investigators using a four-table $\chi^{2}$ test. $\mathrm{P}<0.01$ was considered to indicate a statistically significant difference.

Report interpretation. After obtaining credible analysis data, the report was interpreted. According to the pathogenicity of the pathogen, they were divided into three tiers. The interpretation standards were: i) High-level pathogenic pathogens were reported immediately after detection, such as Mycobacterium tuberculosis; ii) If $\geq 3$ medium-level conditional pathogenic bacteria were identified, the result was reported; iii) Low-level conditional pathogenic bacteria/common colonizing bacteria, such as coagulase-negative staphylococcus, were reported as suspected background microorganisms, and were carefully considered in conjunction with other clinical tests.

For the rare pathogens that were detected, intracellular bacteria and other special pathogens, it was necessary to review the results of biological information analysis and confirm the experimental process, and then report in combination with the clinical history and clinical communication. The reporting rules referred to high-level pathogens for reporting (33).

\section{Results}

Clinical information of patients. The clinical information of the 30 patients is shown in Table I. The results showed 22 out of the 30 patients $(66.67 \%)$ reported a fever, and the majority of them had cough $(23 / 30,76.67 \%)$ and produced sputum $(25 / 30,83.33 \%)$. A total of 10 patients were complicated with a cerebrovascular disease. All patients had consolidation on chest imaging, and 13 patients had pleural effusion
Table I. General clinicopathological information of the recruited patients.

\begin{tabular}{|c|c|}
\hline Parameter & Result \\
\hline $\begin{array}{l}\text { Time from onset to hospital stay, } \\
\text { days }^{\text {a }} \text { (range) }\end{array}$ & $10.71 \pm 18.98(0.5-90)$ \\
\hline $\begin{array}{l}\text { Length of hospital stay, days }{ }^{\mathrm{a}} \\
\text { (range) }\end{array}$ & $14.38 \pm 9.65(5-43)$ \\
\hline Patients admitted to the ICU, $\mathrm{n}$ & 3 \\
\hline $\begin{array}{l}\text { Anti-infection course of treatment, } \\
\text { days }^{\text {a }}\end{array}$ & $13.14 \pm 8.56$ \\
\hline Antifungal course, days ${ }^{\mathrm{a}}$ & $8.59 \pm 22.91$ \\
\hline $\begin{array}{l}\text { Noninvasive ventilatory } \\
\text { support, } \mathrm{n}\end{array}$ & 4 \\
\hline $\begin{array}{l}\text { Noninvasive ventilatory support } \\
\text { duration, days }{ }^{\mathrm{a}}\end{array}$ & $6.75 \pm 4.19$ \\
\hline \multicolumn{2}{|l|}{ Symptoms, $\mathrm{n}$} \\
\hline Fever & 20 \\
\hline Temperature $^{\mathrm{b}}$ & 24 \\
\hline Cough & 23 \\
\hline Expectorant & 25 \\
\hline Chest pain & 3 \\
\hline Chest tightness & 13 \\
\hline Dyspnea & 11 \\
\hline \multicolumn{2}{|l|}{ Complications, $\mathrm{n}$} \\
\hline COPD & 1 \\
\hline Bronchiectasis & 1 \\
\hline Lung cancer & 1 \\
\hline Kidney disease & 1 \\
\hline Cerebrovascular disease & 10 \\
\hline Diabetes & 2 \\
\hline Alzheimer's disease & 1 \\
\hline
\end{tabular}

Routine blood test ${ }^{\mathrm{a}}$

White blood cell count,

$10.88 \pm 5.79(3.4-30.7)$

$10^{9} / 1$ (range)

Neutrophils, $10^{9} / 1$ (range)

$8.48 \pm 6.06(0.43-29.79)$

$214.89 \pm 84.4(82-475)$

Platelets, $10^{9} / 1$ (range)

$50.39 \pm 54.06(5-200)$

C-Reactive protein, mg/l (range)

$1.62 \pm 6.14(0.05-33.27)$

Blood gas analysis, $\%^{\mathrm{a}}$

$\mathrm{PO}_{2}$

$64.81 \pm 19.71(28.2-99.7)$

$\mathrm{PCO}_{2}$

$35.34 \pm 8.14(22.6-55.3)$

$\mathrm{SO}_{2} \%$

$88.64 \pm 12.89(53.2-97.3)$

$\mathrm{pH}$

$7.43 \pm 0.05(7.35-7.51)$

X-ray film, n

Consolidation

30

Consolidation with bronchial signs

Consolidation without bronchial

10

signs

Hydrothorax phenotype

Routine etiology examination

of bacterial culture positive

Fungal positive
13

11

3

${ }^{\mathrm{a}}$ Mean \pm standard deviation; ${ }^{\mathrm{b}}$ within a range of $38.5-41^{\circ} \mathrm{C}$. 


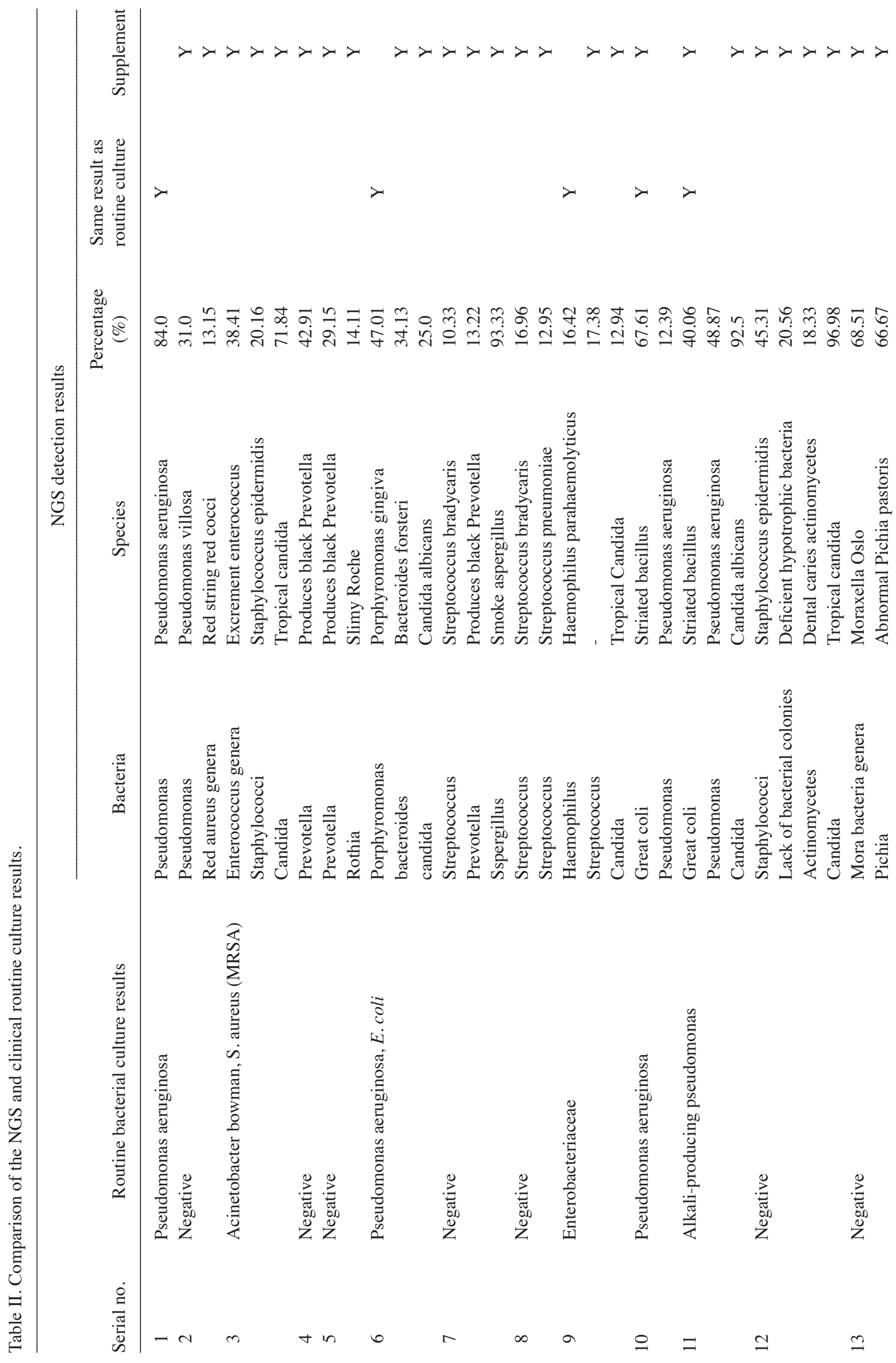




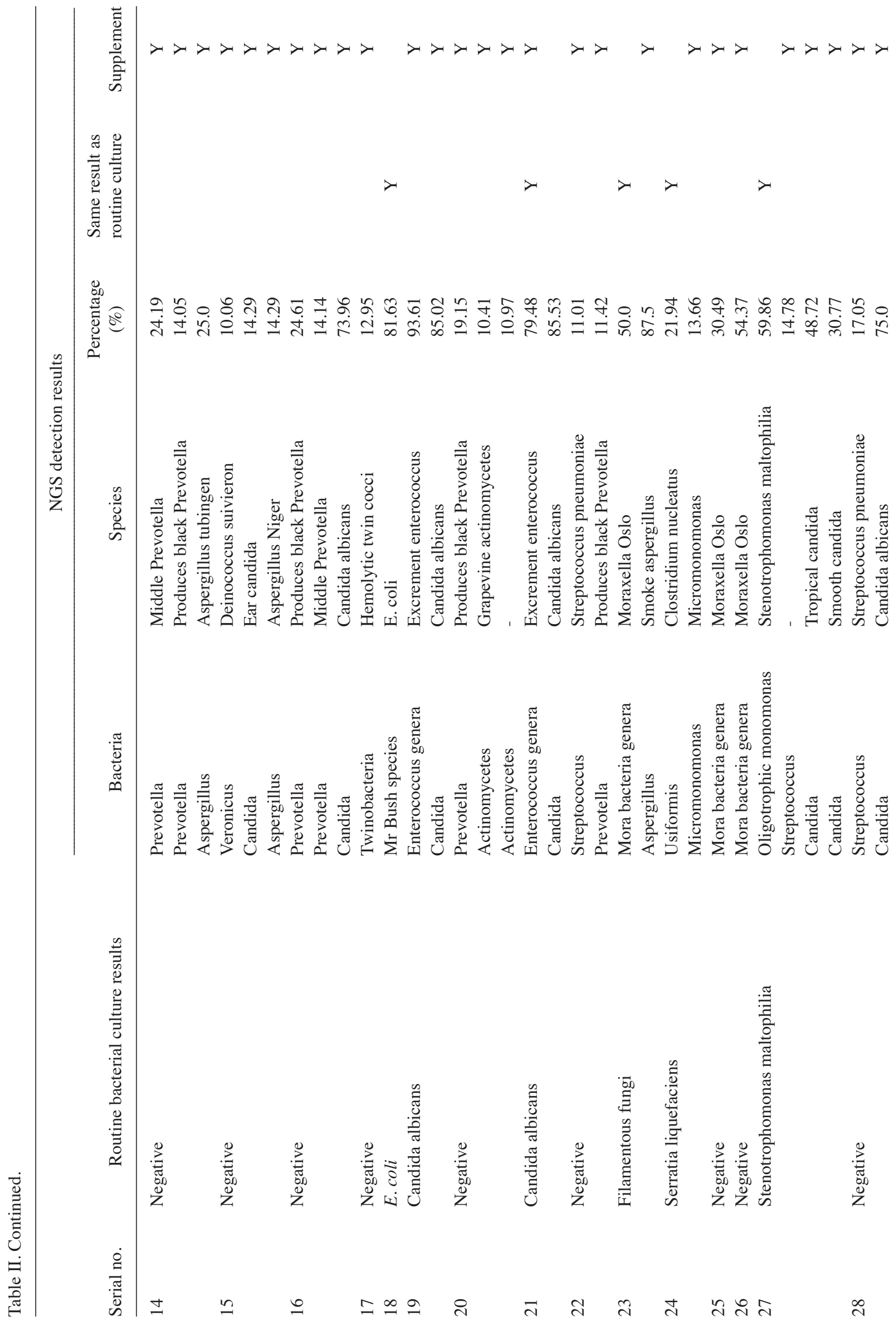


(43.33\%). The results of bacterial culture were positive in only 11 patients $(36.67 \%)$ in routine etiological examination. Fungus was detected in the lavage fluid in 3 patients (10\%). Bacterial culture results of the lavage fluid of 16 patients were negative (53.33\%).

NGS data availability. The microbial gene sequence data obtained were uploaded to the European Nucleotide Archive (ebi.ac.uk/ena). The primary accession no. is PRJEB46374 and the secondary accession no. is ERP130557. The data is publicly available.

Comparison of bacterial culture and NGS detection results of the lavage solution. The comparison of NGS test results and clinical routine culture results of 30 patients is shown in Table II. The results showed that the routine bacterial culture results of the lavage fluid of 16 of the 30 patients were negative (53.3\%), and the remaining 14 were positive (46.6\%). Lavage NGS detection showed significantly more positive results than the conventional bacterial culture, with a $100 \%$ positive rate. Out of those results, 12 of the 30 patients (40\%) had test results that were in complete accordance with the hospital routine bacterial culture results, with 56 mixed infections of pathogenic microorganisms detected. The NGS diagnosis was of great significance to the clinical management of the patients, and all cases were effectively treated to a $100 \%$ recovery rate.

\section{Discussion}

In the present study, the alveolar lavage fluid of 30 patients with a pulmonary infection was tested by routine bacterial culture and NGS, and the results of pathogen detection were compared. All 30 patients showed lung consolidation in the X-ray film (Table I), and tested positive with NGS, whereas the conventional bacterial culture method provided positive results in 14 patients only (46.6\%), with the remaining 16 patients $(53.3 \%)$ being negative. This reflects the serious restrictions of the current diagnostic standards for infectious diseases in the clinic, where only routine bacterial culture is used to determine the infection. In 12 cases, NGS test results were in complete accordance with the bacterial culture results, indicating that in those double positive cases, NGS is compatible to the bacterial culture method. In addition, NGS detected 56 mixed infections of pathogenic microorganism, which had important guiding significance to the clinical diagnosis and treatment, compensating for the deficiency of the conventional bacteriology inspections. The routine bacterial culture method is still important as it can distinguish live bacteria from dead ones (34). However, there are a few possible reasons for the lower microbial detection rates: i) The sampling and transition conditions may cause some of the bacteria to die, thus no detection and ii) antibiotic use before sampling may kill some of the bacteria. The handling errors can be minimised through standardisation of the procedures and increased training for personnel. Antibiotic administration should be avoided before sampling, and if it has been used before sampling, then the use can be noted in the patient's medical history, so that this would be accounted for when analysing the final microbial culture results. Conversely, although NGS cannot distinguish between dead and live bacteria, it is still an important additional tool 
worth exploring, as it does provide additional information and thorough pathological investigation. The results from the NGS performed in the present study are consistent with the Shaanxi Province local etiology epidemic spectrum (35).

These results were in line with the previous literature, confirming that NGS detection can improve the positive rate of pathogen detection and provide guidance for treatment in clinical practice. Li et al (36) performed NGS detection on BALF samples from 32 patients, and the positive detection rate of NGS reached $100 \%$. Compared with the culture method, the diagnostic sensitivity and specificity of NGS were 88.89 and $74.07 \%$, respectively. Cummings et al (37) indicated that for complex microbial specimens, bacterial culture may not be able to identify specific microorganisms, but NGS could detect a wider array of organisms than any other single method alone. A retrospective study aiming to evaluate metagenomic NGS (mNGS) for the clinical diagnosis and prognosis of acute respiratory distress syndrome (ARDS) caused by severe pneumonia, reported that the total positive rate detected by NGS method $(91.1 \%)$ was significantly higher than that detected by the culture method $(62.2 \%)$. The researchers concluded that, mNGS is a valuable tool to determine the etiological value of ARDS caused by severe pneumonia, providing more diagnostic evidence that leads to improving diagnostic accuracy and prognosis for this disease (38). An additional advantage of using NGS is that it identifies anaerobic bacteria that are not usually searched for by clinical laboratories (39).

Early usage of NGS has proven to positively impact the mortality rate as proven by Zhang et al (38). After adjusting the treatment regimen based on the microbiological test results, the 28-day mortality rate in the NGS group (composed of patients tested using NGS and conventional microbiological tests) was significantly lower than that in the non-NGS group (composed of patients given conventional microbiological tests only). NGS significantly reduced the length of stay in the ICU of immunosuppressed patients, shortened the duration of ventilation and reduced the cost of ICU hospitalization $(\mathrm{P}<0.05)(38)$.

In a recent study, comparing NGS with traditional pathogen detection methods in the diagnosis of peripheral pulmonary infectious lesions, NGS could detect and identify a large variety of pathogens, with identification of at least one microbial species in $89 \%$ of the patients with a pulmonary infection. The accuracy and sensitivity of NGS surpassed those of traditional pathogen detection methods. NGS also detected microbes related to human diseases in $94.5 \%$ of samples from patients with pulmonary infection who tested negative when assessed by traditional pathogenic detection methods (40).

As NGS is an accurate and complete method for detection of all the DNA information in a sample, it can be applied to the detection of various pathogenic microorganisms, including bacteria, fungi and viruses $(41,42)$. It can quickly diagnose Acinetobacter baumannii infection (43), directly detect and confirm pneumocystis from clinical specimens (44) and has the ability to detect a variety of fungi (45). Chen et al (46) applied NGS technology to diagnose 9 cases of severe parrot fever pneumonia, and the patients were treated in a timely manner. The results of a prospective multicenter study showed that among 329 adults with severe community-acquired pneumonia, the detection rate of NGS was $90.3 \%$ (47). Cumming et al (37) indicated that for complex microbial specimens, bacterial culture may not be able to identify specific microorganisms, but NGS could detect a greater variety of organisms than any single method alone. This has also been confirmed in other studies that have shown that NGS is considerably more sensitive in the diagnosis of mixed pulmonary infection than conventional methods $(97.2 \%$ vs. $13.9 \%)(40,48,49)$. Furthermore, NGS has been proposed as an important auxiliary diagnostic method for rare pathogens (50). Currently, the new corona pneumonia is also diagnosed by NGS, which can complement and improve clinical pathogenic diagnosis (51). Therefore, NGS is a good pathogenic monitoring method and is worthy of extended application in clinical settings.

For pneumonia patients who are not suitable for bronchoalveolar lavage, the NGS test of tracheal secretions can also provide ideal results (52). A recent study suggested that for bacteria and fungi detection, BALF NGS is more sensitive than blood NGS, whereas blood NGS can identify the pathogens of pneumonia, especially for some viruses (53). It is worth mentioning that NGS enabled complete sequencing of the long SARS-CoV-2 genome ( 30,000 nucleotides). NGS has also provided insight into strain origin and viral evolution (54). The extensive application of NGS will provide powerful support in the fight against future public health emergencies. The application of these sequencing techniques could further benefit SARS-CoV-2 diagnostic development and novel therapeutic target discovery, as well as blocking interspecies transmission by identifying intermediate SARS-CoV-2 novel hosts (53).

The present study has local significance, providing new information regarding the pathogens present in patients with a lung infection in the Xi'an region. NGS has been used in several studies in Xi'an region for detection of pathogens in various infections. Hu et al (55) used NGS to detect SARS-CoV-2 in eye swabs. Lin et al (56) used NGS and a metagenomic approach to detect and characterize respiratory bacteria in an unexplained pneumonia case in an infant. Yang et al (57) used NGS to accurately diagnose granulomatous amoebic encephalitis caused by Balamuthia mandrillaris in a child. Li et al (58) used NGS along with electromagnetic navigation bronchoscopy to diagnose Mycobacterium chelonae. Xu et al (59) used NGS to characterize lncRNAs in 293T cells after human foamy virus infection. No comprehensive study has been performed to compare the detection of bacteria using NGS and routine culture. To the best of our knowledge, the present study is the first in the region, and may provide valuable initial data for future comparative and/or epidemiological studies.

In conclusion, early detection of pathogens can optimize antibiotic selection, and consequently improve survival rates in pulmonary infection. NGS is a new technology that uses high-throughput sequencing to sequence all the DNA in human samples. Subsequent quantitative and bioinformatics analyses of microbial species in the samples can provide a reliable basis for clinical diagnosis and treatment. The results of the present study show the prevalence of respiratory pathogens in Xi'an region and supports the implementation of NGS in clinical routine diagnosis for pathogen identification, especially when the results of routine bacterial cultures are negative. Timely sequencing in addition to routine bacterial culture testing will further assist in improving the accuracy of diagnosis and in administration of a more accurate antibiotic regimen. 


\section{Acknowledgements}

Not applicable.

\section{Funding}

This research was funded by Shaanxi Provincial Key Research and Development Program (Shaanxi, China; grant no. 2019SF-043) and New Zealand Ministry of Education, China-New Zealand Tripartite Research Collaboration Fund (grant no. AUT-13772).

\section{Availability of data and materials}

The datasets used and/or analyzed during the present study are available from the corresponding author on reasonable request.

\section{Authors' contributions}

JS and JL conceived the study. YW, RZ, LZ, RL, MC, CL XW, MZ, PW and SY designed the study. YW, JC, RZ and LZ validated the study. AH, JC, JL and YW analyzed the data. YW, RZ, LZ, RL, MC, CL, XW, MZ, PW and SY performed the investigations. JS, YW, RZ, LZ, RL, MC, CL, XW, MZ, PW and SY curated the data. AH, JS, YW and RZ drafted the manuscript. JL reviewed and edited the manuscript. JS, JC, YW and RZ confirm the authenticity of all the raw data. All authors have read and approved the final manuscript.

\section{Ethics approval and consent to participate}

The study was performed in accordance with the guidelines described in the Declaration of Helsinki and approved by the Norinco 521 Hospital Ethics Committee (Xi'an, China; approval no. 201901) on 9 January 2019.

\section{Patient consent for publication}

Informed consent was obtained from all subjects involved in the study.

\section{Competing interests}

The authors declare that they have no competing interests.

\section{References}

1. Walker CLF, Rudan I, Liu L, Nair H, Theodoratou E, Bhutta ZA, O'Brien KL, Campbell H and Black RE: Global burden of childhood Pneumonia and Diarrhoea. Lancet 381: 1405-1416, 2013.

2. Reza MB, Lotfian F, Aghahosseini F, Cheraghvandi A, Khalilzadeh S, Farjah A and Boloursaz M: Epidemiology of lower respiratory tract infections in children. J Compr Ped 3: 93-98, 2013.

3. GBD 2015 LRI Collaborators: Estimates of the global, regional, and national morbidity, mortality, and aetiologies of lower respiratory tract infections in 195 countries: A systematic analysis for the Global Burden of Disease Study 2015. Lancet Infect Dis 17: 1133-1161, 2017.

4. Mahendra M, Jayaraj BS, Lokesh KS, Chaya SK, Veerapaneni VV, Limaye S, Dhar R, Swarnakar R, Ambalkar S and Mahesh PA Antibiotic prescription, organisms and Its resistance pattern in patients admitted to respiratory ICU with respiratory infection in Mysuru. Indian J Crit Care Med 22: 223-230, 2018.
5. Trenholme AA, Byrnes CA, McBride C, Lennon DR, Chan-Mow F, Vogel AM, Stewart JM and Percival T: Respiratory health outcomes 1 year after admission with severe lower respiratory tract infection. Pediatr Pulmonol 48: 772-779, 2013.

6. Nathan AM, The CSJ, Eg KP, Jabar KA, Zaki R, Hng SY, Thavagnanam $\mathrm{S}$ and de Bruyne JA: Respiratory sequelae and quality of life in children one-year after being admitted with a lower respiratory tract infection: A prospective cohort study from a developing country. Pediatr Pulmonol 55: 407-417, 2019.

7. Worrall G: Bronchiolitis. Can Fam Physician 54: 742-743, 2008.

8. World Health Organization (WHO): The top ten cause of death factsheet. WHO, Geneva, 2020. http://www.who.int/mediacentre/factsheets/fs310/en/index.html. Accessed December 9, 2020.

9. Welte T, Torres A and Nathwani D: Clinical and economic burden of community-acquired pneumonia among adults in Europe. Thorax 67: 71-79, 2011.

10. Mani CS and Murray DL: Acute pneumonia and its complications. Prin Prac Ped Inf Dis 235-245. e4, 2012.

11. Jain S, Self WH, Wunderink RG, Fakhran S, Balk R, Bramley AM, Reed C, Grijalva CG, Anderson EJ, Courtney DM, et al: Community-acquired pneumonia requiring hospitalization among U.S. adults. N Engl J Med 373: 415-427, 2015.

12. Gadsby NJ, Russell CD, McHugh MP, Mark H, Conway Morris A, Laurenson IF, Hill AT and Templeton KE: Comprehensive molecular testing for respiratory pathogens in community-acquired pneumonia. Clin Infect Dis 62: 817-823, 2016.

13. American Thoracic Society,Infectious Disease Society of America: Guidelines for the management of adults with hospital-acquired, ventilator-associated, and healthcare-associated pneumonia: Am J Respir Crit Cre Med 171: 388-416, 2005.

14. Mandell LA, Bartlett JG, Dowell SF, File TM Jr, Musher DM and Whitney C; Infectious diseases society of America: Update of practice guidelines for the management of community-acquired pneumonia in immunocompetent adults. Clin Infect Dis 37: 1405-1433, 2003.

15. Takeuchi S, Kawada JI, Horiba K, Okuno Y, Okumura T, Suzuki T, Torii Y, Kawabe S, Wada S, Ikeyama T and Ito Y: Metagenomic analysis using next-generation sequencing of pathogens in bronchoalveolar lavage fluid from pediatric patients with respiratory failure. Sci Rep 9: 12909, 2019.

16. Hohenthal U, Itala M, Salonen J, Sipila J, Rantakokko-Jalava K, Meurman O, Nikoskelainen J, Vainionpaa R and Kotilainen P: Bronchoalveolar lavage in immunocompromised patients with haematological malignancy-value of new microbiological methods. Eur J Haematol 74: 203-211, 2005.

17. Bewig B, Haacke TC, Tiroke A, Bastian A, Böttcher H, Hirt SW, Rautenberg P and Haverich A: Detection of CMV pneumonitis after lung transplantation using PCR of DNA from bronchoalveolar lavage cells. Respiration 67: 166-172, 2000.

18. Nadimpalli S, Foca M, Satwani P, Sulis ML, Constantinescu A and Saiman L: Diagnostic yield of bronchoalveolar lavage in immunocompromised children with malignant and non-malignant disorders. Pediatr Pulmonol 52: 820-826, 2017.

19. Rao U, Piccin A, Malone A, O'Hanlon K, Breatnach F, O'Meara A McDermott M, Butler K, O'Sullivan N, Russell J, et al: Utility of bronchoalveolar lavage in the diagnosis of pulmonary infection in children with haematological malignancies. Ir J Med Sci 182: 177-183, 2012.

20. Efrati O, Gonik U, Bielorai B, Modan-Moses D, Neumann Y, Szeinberg A, Vardi A, Barak A, Paret G and Toren A: Fiberoptic bronchoscopy and bronchoalveolar lavage for the evaluation of pulmonary disease in children with primary immunodeficiency and cancer. Pediatr Blood Cancer 48: 324-329, 2007.

21. Harris AM, Hicks LA and Qaseem A; High Value Care Task Force of the American College of Physicians and for the Centers for Disease Control and Prevention: Appropriate antibiotic use for acute respiratory tract infection in adults: Advice for high-value care from the American college of physicians and the centers for disease control and prevention. Ann Intern Med 164: 425-434, 2016

22. Duan N, Du J, Huang C and Li H: Microbial distribution and antibiotic susceptibility of lower respiratory tract infections patients from pediatric ward, adult respiratory ward, and respiratory intensive care unit. Front Microbiol 11: 1480, 2020.

23. Schlaberg R, Queen K, Simmon K, Tardif K, Stockmann C, Flygare S, Kennedy B, Voelkerding K, Bramley A, Zhang J, et al: Viral pathogen detection by metagenomics and pan-viral group polymerase chain reaction in children with pneumonia lacking identifiable etiology. J Infect Dis 215: 1407-1415, 2017. 
24. Houldcroft CJ, Beale MA and Breuer J: Clinical and biological insights from viral genome sequencing. Nat Rev Microbiol 15: 183-192, 2017.

25. Wilson MR, Naccache SN, Samayoa E, Biagtan M, Bashir H, Yu G, Salamat SM, Somasekar S, Federman S, Miller S, et al Actionable diagnosis of neuroleptospirosis by next-generation sequencing. N Engl J Med 370: 2408-2417, 2014.

26. Graf EH, Simmon KE, Tardif KD, Hymas W, Flygare S, Eilbeck K, Yandell M and Schlaberg R: Unbiased detection of respiratory viruses by use of RNA sequencing-based metagenomics: A systematic comparison to a commercial PCR panel J Clin Microbiol 54: 1000-1007, 2016.

27. Thorburn F, Bennett S, Modha S, Murdoch D, Gunson R and Murcia PR: The use of next generation sequencing in the diagnosis and typing of respiratory infections. J Clin Virol 69: 96-100,2015.

28. Kawada J, Okuno Y, Torii Y, Okada R, Hayano S, Ando S, Kamiya Y, Kojima S and Ito Y: Identification of viruses in cases of pediatric acute encephalitis and encephalopathy using next-generation sequencing. Sci Rep 6: 33452, 2016

29. Suzuki T, Kawada JI, Okuno Y, Hayano S, Horiba K, Torii Y, Takahashi Y, Umetsu S, Sogo T, Inui A and Ito Y: Comprehensive detection of viruses in pediatric patients with acute liver failure using next-generation sequencing. J Clin Virol 96: 67-72, 2017.

30. Horiba K, Kawada JI, Okuno Y, Tetsuka N, Suzuki T, Ando S, Kamiya Y, Torii Y, Yagi T, Takahashi Y and Ito Y: Comprehensive detection of pathogens in immunocompromised children with bloodstream infections by next-generation sequencing. Sci Rep 8: 3784, 2018.

31. Takeuchi S, Kawada JI, Okuno Y, Horiba K, Suzuki T, Torii Y, Yasuda K, Numaguchi A, Kato T, Takahashi Y and Ito Y: Identification of potential pathogenic viruses in patients with acute myocarditis using next-generation sequencing. J Med Virol 90: 1814-1821, 2018.

32. Miao Q, Ma Y, Wang Q, Pan J, Zhang Y, Jin W, Yao Y, Su Y, Huang Y, Wang M, et al: Microbiological diagnostic performance of metagenomic next-generation sequencing when applied to clinical practice. Clin Infect Dis 67 (Suppl 2): S231-S240, 2018.

33. Chinese Society of Laboratory Medicine: Expert consensus on the standardized management of bioinformatics analysis for the detection of pathogenic microorganisms in mNGS. Chin J Lab Med 44: 799-807, 2021 (In Chinese). DOI: 10.3760/ cma.j.cn114452-20210322-00178

34. Lagier JC, Edouard S, Pagnier I, Mediannikov O, Drancourt M and Raoult D: Current and past strategies for bacterial culture in clinical microbiology. Clin Microbiol Rev 28: 208-236. 2015.

35. Zhang YL: Analysis of the distribution and drug resistance of pathogenic bacteria in hospitalized infected patients in our hospital from 2015 to 2018. Clin Res Pract 4: 123-125. 2019 (In Chinese). DOI: 10.19347/j.cnki.2096-1413.201927054

36. Li Y, Sun B, Tang X, Liu YL, He HY, Li XY, Wang R, Guo F and Tong $\mathrm{ZH}$ : Application of metagenomic next-generation sequencing for bronchoalveolar lavage diagnostics in critically ill patients. Eur J Clin Microbiol Infect Dis 39: 369-374, 2020.

37. Cummings LA, Hoogestraat DR, Rassoulian-Barrett SL, Rosenthal CA, Salipante SJ, Cookson BT and Hoffman NG: Comprehensive evaluation of complex polymicrobial specimens using next generation sequencing and standard microbiological culture. Sci Rep 10: 5446, 2020.

38. Zhang P, Chen Y, Li S, Li C, Zhang S, Zheng W, Chen Y, Ma J, Zhang X, Huang Y and Liu S: Metagenomic next-generation sequencing for the clinical diagnosis and prognosis of acute respiratory distress syndrome caused by severe pneumonia: A retrospective study. PeerJ 8: e9623, 2020.

39. Leo S, Gaïa N, Ruppé E, Emonet S, Girard M, Lazarevic V and Schrenzel J: Detection of Bacterial Pathogens from Broncho-Alveolar Lavage by Next-Generation sequencing. Int J Mol Sci 18: 2011, 2017.

40. Huang J, Jiang E, Yang D, Wei J, Zhao M, Feng J and Cao J: Metagenomic next-generation sequencing versus traditional pathogen detection in the diagnosis of peripheral pulmonary infectious lesions. Infect Drug Resist 13: 567-576, 2020.

41. Jianchao J, Jianmin J and Zi L: Value of metagenomics next generation sequencing to the diagnosis of fungal infection in patients with severe pneumonia. J Chin Pract Diagn 34: 1023-1025, 2020 DOI number DOI: 10.13507/j.issn.1674-3474.2020.10.014

42. Shiqing Y, Jie Y and Chaochao L: The diagnostic value of respiratory aetiology gene detection in pulmonary alveolar lavage fluid in refractory lower respiratory tract infection. Mod Med Health Res 3: 85-87, 2019. https://kns.cnki.net/kcms/detail/detail.aspx?dbcode $=$ CJFD \&dbname $=$ CJFDLAST2020\&filename $=$ XYJD2 01914041\&uniplatform $=$ NZKPT\&v=EvKaKS8OMLbeS66kDF O8oBsldHJlcHgi8g9rEE5vAb8-TbAWSaYLv7gz2muZlRdf
43. Xu A, Zhu H, Gao B, Weng H, Ding Z, Li M, Weng X and He G: Diagnosis of severe community-acquired pneumonia caused by Acinetobacter baumannii through next-generation sequencing: A case report. BMC Infect Dis 20: 45, 2020.

44. Irinyi L, Hu Y, Hoang MTV, Pasic L, Halliday C, Jayawardena M, Basu I, McKinney W, Morris AJ, Rathjen J, et al: Long-read sequencing based clinical metagenomics for the detection and confirmation of Pneumocystis jirovecii directly from clinical specimens: A paradigm shift in mycological diagnostics. Med Mycol 58: 650-660,2020.

45. McTaggart LR, Copeland JK, Surendra A, Wang PW, Husain S, Coburn B, Guttman DS and Kus JV: Mycobiome sequencing and analysis applied to fungal community profiling of the lower respiratory tract during fungal pathogenesis. Front Microbiol 10: 512,2019

46. Chen X, Cao K, Wei Y, Qian Y, Liang J, Dong D, Tang J, Zhu Z, $\mathrm{Gu} \mathrm{Q}$ and $\mathrm{Yu} \mathrm{W}$ : Metagenomic next-generation sequencing in the diagnosis of severe pneumonias caused by Chlamydia psittaci. Infection 48: 535-542, 2020.

47. Wu X, Li Y, Zhang M, Li M, Zhang R, Lu X, Gao W, Li Q, Xia Y, Pan P and Li Q: Etiology of severe community-acquired pneumonia in adults based on metagenomic next-generation sequencing: A prospective multicenter study. Infect Dis Ther 9: $1003-1015,2020$

48. Wang J, Han Y and Feng J: Metagenomic next-generation sequencing for mixed pulmonary infection diagnosis. BMC Pulm Med 19: 252, 2019.

49. Fang X, Mei Q, Fan X, Zhu C, Yang T, Zhang L, Geng S and Pan A: Diagnostic value of metagenomic next-generation sequencing for the detection of pathogens in bronchoalveolar lavage fluid in ventilator-associated pneumonia patients. Front Microbiol 11: 599756, 2020.

50. Xiao W, Han P, Xu Z and Huang M: Pulmonary scedosporiosis in a patient with acute hematopoietic failure: Diagnosis aided by next-generation sequencing. Int J Infect Dis 85: 114-116, 2019.

51. Liu S, Li Q, Chu X, Zeng M, Liu M, He X, Zou H, Zheng J, Corpe C, Zhang X, et al: Monitoring coronavirus disease 2019: A review of available diagnostic tools. Front Public Health 9: 672215,2021

52. Kalantar KL, Moazed F, Christenson SC, Wilson J, Deiss T, Belzer A, Vessel K, Caldera S, Jauregui A, Bolourchi S, et al: Metagenomic comparison of tracheal aspirate and mini-bronchial alveolar lavage for assessment of respiratory microbiota. Am J Physiol Lung Cell Mol Physiol 316: L578-L584, 2019.

53. Chen X, Ding S, Lei C, Qin J, Guo T, Yang D, Yang M, Qing J, $\mathrm{He} \mathrm{W}$, Song M, et al: Blood and bronchoalveolar lavage fluid metagenomic next-generation sequencing in pneumonia. Can J Infect Dis Med Microbiol 2020: 6839103, 2020

54. D'Cruz RJ, Currier AW and Sampson VB: Laboratory testing methods for novel severe acute respiratory syndromecoronavirus-2 (SARS-CoV-2). Front Cell Dev Biol 8: 468, 2020.

55. Hu Y, Chen T, Liu M, Zhang L, Wang F, Zhao S, Liu H, Xia H, Wang Y and Li L: Positive detection of SARS-CoV-2 combined HSV1 and HHV6B virus nucleic acid in tear and conjunctival secretions of a non-conjunctivitis COVID-19 patient with obstruction of common lacrimal duct. Acta Ophthalmol 98: 859-863, 2020

56. Lin Y, Wang BX, Zhang NN, Zhang L, Gao ZB, Tian J and Jiang X: Metagenomic analysis identified Stenotrophomonas maltophilia pneumonia in an infant suffering from unexplained very severe pneumonia. Front Pediatr 7: 380, 2019.

57. Yang Y, Hu X, Min L, Dong X and Guan Y: Balamuthia mandrillaris-related primary amoebic encephalitis in China diagnosed by next generation sequencing and a review of the literature. Lab Med 51: e20-e26, 2020

58. Li Y, Zang Y, Wang Y, Jin F and Liu W: Peripheral pulmonary nodule diagnosed as mycobacterium chelonae using electromagnetic navigation bronchoscopy combined with next generation sequencing: A case report. Am J Transl Res 12: 4066-4073. 2020.

59. Xu S, Dong L, Shi Y, Chen L, Yuan P, Wang S, Li Z, Sun Y, Han S, Yin J, et al: The novel landscape of long non-coding RNAs in response to human foamy virus infection characterized by RNA-Seq. AIDS Res Hum Retroviruses 33: 452-464, 2017.

This work is licensed under a Creative Commons Attribution-NonCommercial-NoDerivatives 4.0 International (CC BY-NC-ND 4.0) License. 